\title{
Treatment resistant Lyme arthritis caused by Borrelia garinii
}

\author{
F-X Limbach, B Jaulhac, X Puechal, H Monteil, J-L Kuntz, Y Piemont, J Sibilia
}

\begin{abstract}
Lyme arthritis is caused in Europe by three main pathogenic species of Borrelia burgdorferi sensu lato: Borrelia burgdorferi sensu stricto, Borrelia garinii, and Borrelia afzelii. Because few synovial samples have yet been analysed by species-specific DNA amplification methods, further studies are needed to define the spectra of clinical manifestations associated with these different species. Two cases of treatment resistant Lyme arthritis are reported here, in which DNA amplification of the flagellin gene followed by dot-blot hybridisation in the synovial fluid identified $B$ garinii as the causative agent. Clinical and biological data did not differ from the usual descriptions of Lyme arthritis, but as the recently reported molecular mimicry between OspA and hLFA1 is not applicable to $B$ garinii, the pathogenesis of the present cases remains unclear. Future studies should aim at assessing the role of $B$ garinii in European Lyme arthritis and its possible pathogenic and therapeutic consequences.

(Ann Rheum Dis 2001;60:284-286)
\end{abstract}

Lyme borreliosis is a multisystemic disease caused by the spirochaete Borrelia burgdorferi (B burgdorferi) sensu lato. On the basis of DNA similarities, three principal pathogenic species of $B$ burgdorferi sensu lato have been identified, $B$ burgdorferi sensu stricto, $B$ afzelii, and $B$ garinii. Lyme arthritis would seem to be the most commonly disseminated form of the disease in the USA, whereas late cutaneous manifestations of Lyme borreliosis occur almost exclusively in Europe. This observation, together with the fact that although all three pathogenic species are present in Europe only B burgdorferi sensu stricto has been isolated in North America, has led to the hypothesis that the tropism of the different species of $B$ burgdorferi sensu lato can be either articular, cutaneous, or neurological. Because it has been reported that patient serum samples might react more strongly with heterologous than with homologous strains, ${ }^{1}$ serological methods are not suitable to assess this correlation, and culture techniques likewise cannot be applied to synovial tissue or fluid. Hence the only reliable methods for typing Borrelia strains in synovial samples are based on DNA amplification. When such direct identification methods were used, acrodermatitis chronica atrophicans and neuroborreliosis were found to be mainly associated with $B$ afzelii ${ }^{2}$ and $B$ garinii, ${ }^{3}$ respectively.
Recently, four European groups ${ }^{4-7}$ and our own ${ }^{8}$ identified the Borrelia species involved in some forms of Lyme arthritis. A variety of identification techniques were used, including DNA sequencing of OspA, ${ }^{4}$ OspA speciesspecific primers, ${ }^{5}$ and reverse line blotting ${ }^{67}$ of synovial fluid. Discordant results were obtained, particularly concerning the role of $B$ garinii, while the clinical presentation and outcome of patients were not described in detail. Furthermore, because only a few synovial fluid samples were analysed by species-specific DNA amplification methods, studies are still needed to determine which spectrum of clinical manifestations is associated with $B$ garinii. In the present work we describe two patients with treatment resistant Lyme arthritis, in whom DNA amplification and dot-blot hybridisation identified $B$ garinii as the causative species.

\section{Case reports}

PATIENT 1

Patient 1, a 41 year old woman, presented in December 1993 with a history of monarthritis of the right knee. This had developed in August 1991, a few months after the disappearance of a concentric erythematous eruption on the calf. She could not recall having been bitten by a tick during her frequent forest walks near Paris (France). The right knee swelling had been treated several times between August 1991 and January 1993 with corticosteroid infiltrations, but this treatment had never been effective for more than six months. The patient had no history of psoriasis, low back pain, or genital infection.

On examination at the rheumatology department the right knee was found to be swollen, though with minimal pain. The synovial fluid contained $28 \times 10^{9}$ leucocytes $/ 1$ (64\% polymorphonuclear cells and 36\% lymphocytes), while a synovial biopsy specimen showed a dense inflammatory infiltrate of lymphocytes and plasmocytes. Tests for antinuclear antibodies and rheumatoid factor were negative and the patient's haplotype was HLA-B18/40 and DRB ${ }^{\star} 0403 /{ }^{\star} 0404$. Radiographs of the right knee showed demineralisation without any erosion. Chlamydia trachomatis serology was negative. A serological enzyme linked immunosorbent assay (ELISA) test for Lyme disease was strongly positive with values of 4.2 for IgG (>1.1 positive) and negative for IgM, which were confirmed by western blot analyses. Because only small amounts of synovial fluid were available, DNA amplification specific for $B$ burgdorferi sensu lato was not interpretable. The patient was treated with doxycycline 
(200 mg/day) for one month, with a transient recovery.

In July 1994 the patient had her first relapse since antibiotic treatment. Puncture of the knee yielded $10 \mathrm{ml}$ of fluid containing $10.4 \times$ $10^{9}$ leucocytes $/ 1$ ( $21 \%$ polymorphonuclear cells and $68 \%$ lymphocytes), while serological ELISA tests gave a value of 2.5 for IgG and were negative for IgM. The patient was treated parenterally with ceftriaxone $(2 \mathrm{~g} /$ day $)$ for three weeks. Owing to persistent swelling, triamcinolone hexacetonide $(40 \mathrm{mg})$ was injected into the knee, with successful results.

When painful swelling recurred in January 1995, knee puncture disclosed a synovial fluid containing $7.8 \times 10^{9}$ leucocytes $/ 1$ ( $45 \%$ polymorphonuclear cells and $20 \%$ lymphocytes). In this last fluid, DNA amplification using a polymerase chain reaction (PCR) specific for $B$ burgdorferi sensu lato ${ }^{9}$ was positive. The patient was successfully treated by an intra-articular injection of $10 \mathrm{ml}$ of $1 \%$ osmic acid.

In October 1995 symptoms recurred, and knee puncture yielded $25 \mathrm{ml}$ of inflammatory synovial fluid. A third course of antibiotic treatment was given, ceftriaxone (2 g/day) parenterally for three weeks. There was further swelling in 1996 requiring the removal of $40 \mathrm{ml}$ of synovial fluid $\left(6.2 \times 10^{9}\right.$ leucocytes $/ 1,90 \%$ of which were lymphocytes), and in 1998 still a little swelling of the right knee persisted.

PATIENT 2

Patient 2, a 27 year old man living in the East of France, was referred in February 1998 for arthritis of the left knee. An effusion of the left knee joint, which had developed five months previously, had been treated initially with nonsteroidal anti-inflammatory drugs and, because of a lack of efficacy, with an intra-articular cortivazol injection in November 1997. After a transient improvement, swelling of the left knee had recurred 15 days later. As this patient lives in an endemic area, was frequently bitten by ticks, and had a possible previous history of erythema migrans in September 1996, treatment with amoxicillin (4 g/day orally) was given in December 1997 for three weeks but without effect.

Physical examination showed a marked swelling of the left knee, which was moderately painful. Radiographs of the knees, hands, and sacroiliac joints were normal, and tests for antinuclear antibodies, rheumatoid factor, and Chlamydia trachomatis were negative. The patient's haplotype was HLA-B7/62 and $\mathrm{DRB} 1^{\star} 0101 /{ }^{\star} 11$. Blood tests showed an increase in the acute phase reactants (erythrocyte sedimentation rate $86 \mathrm{~mm} / 1 \mathrm{st} \mathrm{h}, \mathrm{C}$ reactive protein $80 \mathrm{mg} / \mathrm{l}$ ), with a normal white blood cell count. Lyme serology was positive by ELISA with values of $1450 \mathrm{U}$ for IgG ( $>9 \mathrm{U}$ positive) and $41 \mathrm{U}$ for $\operatorname{IgM}(>10 \mathrm{U}$ positive), which were confirmed by western blot analyses. The synovial fluid contained $20 \times 10^{9}$ leucocytes $/ 1$ (80\% polymorphonuclear cells and $20 \%$ lymphocytes) and DNA amplification using a PCR specific for $B$ burgdorferi sensu lato ${ }^{9}$ was positive in this sample. The patient was treated for three weeks with intravenous ceftriaxone (2 g/day) and was completely cured, with no recurrence after an 18 months' follow up.

\section{Methods}

Synovial fluid samples were obtained from each patient by needle puncture. PCR amplification of a central part of the flagellin gene, using as primers two oligonucleotides specific for all B burgdorferi sensu lato species, was performed as previously described ${ }^{9}$ with both synovial fluids and gave positive results. To avoid DNA contamination of samples, precautions previously described ${ }^{9}$ were taken. A species-specific dot-blot hybridisation assay ${ }^{8}$ was then performed on the PCR products. Oligonucleotide probes specific for the following $B$ burgdorferi sensu lato species were used: $B$ burgdorferi sensu stricto, $B$ garinii, $B$ afzelii, $B$ japonica, $B$ andersonii, $B$ valaisiana, and $B$ bissettii. Synovial samples from the two patients hybridised only with the $B$ garinii probe and there was no evidence of mixed infection.

\section{Discussion}

This is the first description of Lyme arthritis caused by $B$ garinii, which was treatment resistant in the first case and amoxicillin resistant in the second, albeit after intra-articular corticosteroids. Although a diagnosis of Lyme arthritis is obvious for the second patient, the history of the first patient is more complicated. However, this diagnosis was retained based on the following data: possible erythema migrans, recurrent monarthritis with a positive serology for B burgdorferi, positive PCR, the specificity of which has previously been shown to be absolute $^{9}$ for $B$ burgdorferi when precautions are taken to avoid cross contamination between samples. No other diagnosis was made after seven years' follow up. It is noteworthy that the clinical manifestations and biological data of these patients did not differ from the usual descriptions of Lyme arthritis. Thus both had a possible history of tick bites and erythema migrans, knee arthritis with marked swelling but minimal pain, and concordant serological data. This uniform clinical presentation of Lyme arthritis, whatever the species involved, differs from the recent reports of patients presenting with erythema migrans, in whom different symptoms were observed according to whether the causative agent was $B$ burgdorferi sensu stricto or $B$ afzelii. ${ }^{10}$ In our two patients, as previously reported in other cases of Lyme arthritis, local corticosteroid treatment resulted in a temporary improvement, but might have been implicated in the resistance to amoxicillin in the second patient. The most striking clinical feature of these two patients was the persistent arthritis for years after several courses of antibiotics, including ceftriaxone twice in the first case and seven weeks after amoxicillin in the second patient. Because $B$ garinii DNA was still detected in synovial fluid two years after the onset of disease despite two courses of antibiotics in the first patient, the chronic persistence of $B$ garinii is likely. However, even if $\mathrm{we}^{9}$ and others ${ }^{11}$ have previously demonstrated the persistence of 
$B$ burgdorferi sensu lato DNA in treatment resistant Lyme arthritis, this does not firmly denote the presence of viable spirochaetes.

Species typing by DNA amplification has recently led to controversial results concerning the prevalence of $B$ garinii in Lyme arthritis. In two German studies, arthritis was found to be caused by $B$ garinii in six of $11^{5}$ and three of seven cases, ${ }^{4}$ and no predominance of $B$ burgdorferi sensu stricto was seen in these patients. Conversely, other German authors ${ }^{7}$ and some of us ${ }^{8}$ identified DNA specific for $B$ garinii only in one of 17 and one of 10 synovial fluid samples respectively, while Dutch authors ${ }^{6}$ detected exclusively $B$ burgdorferi sensu stricto DNA in three articular localisations of Lyme borreliosis. Comparing these different studies, it would appear that Lyme arthritis is primarily due to $B$ burgdorferi sensu stricto and that $B$ garinii only rarely plays a part in the articular manifestations of the disease in Europe.

DRB1 alleles of our patients were DRB $1^{\star} 0404$ and ${ }^{\star} 0101$, which are known to be associated with treatment resistant Lyme arthritis in the USA. ${ }^{12}$ Recently, Gross et al showed that seven of 11 patients with treatment resistant Lyme arthritis had at least one HLA-DRB1 allele associated with susceptibility to rheumatoid arthritis. ${ }^{13}$ These susceptibility alleles share the same epitope at residues 67 to 74 of the HLA third hypervariable region, which could bind the OspA amino acids 165 to 173 of $B$ burgdorferi sensu stricto. ${ }^{13}$ In view of similar homology between the OspA 165-173 amino acids and a peptide of the human leucocyte function associated antigen 1 (hLFA1), hLFA1 has been proposed as a candidate autoantigen to explain the chronic course and treatment resistance of some forms of Lyme arthritis. Our two patients each had one susceptibility allele, but the OspA 165-173 sequence of $B$ garinii is not closely related to that of hLFA1. ${ }^{13}$ Hence this autoimmune mechanism cannot explain the treatment resistance of all patients. Accordingly, in another recent study it was found that even if the duration of disease after antibiotic treatment correlated with the $\mathrm{T}$ cell response to dominant epitopes of OspA in most cases of treatment resistant Lyme arthritis, the $\mathrm{T}$ cells of a small number of patients did not react with OspA. ${ }^{14}$ Alternative mechanisms, including mimicry between other antigens, such as the flagellar protein $\mathrm{P} 41$ of $B$ burgdorferi and a member of the $60 \mathrm{kDa}$ family of human heat shock proteins, ${ }^{14}$ remain to be investigated. It can also be speculated that in addition to the genetic susceptibility of the patient as seen in the USA, the causative species of Borrelia may play a part in the treatment resistance of the disease in Europe. Meanwhile, a comparison in European patients of HLA haplotypes and Borrelia strains in clinical samples would give an indication of whether the organotropism of the spirochaete is at least partially linked to the genetic predisposition of the patient and, in particular, to the third hypervariable region of the DRB1 HLA chain.
In summary, $B$ garinii can be implicated in European Lyme arthritis and gives a clinical presentation similar to that of $B$ burgdorferi sensu stricto. Moreover, Lyme arthritis due to $B$ garinii may be associated in susceptible hosts with amoxicillin resistance or treatment resistance, as in these two patients. The recently described molecular mimicry between OspA and hLFA1 may not be applicable to these patients and future studies should aim at assessing further the role of $B$ garinii in European Lyme arthritis and its possible pathogenic and therapeutic consequences.

The authors are grateful to SE Malawista for helpful discussion and criticism of the manuscript, and to D Herb and C Barthel for excellent technical assistance.

Part of this work was supported by the Programme Hospitalier de Recherche Clinique 1995.

1 Karlsson M. Antibody response against autologous and heterologous isolates of Borrelia burgdorferi in four patients with Lyme neuroborreliosis. Eur J Clin Microbiol Infect Dis 1991;10:742-5.

2 Picken RN, Strle F, Picken MM, Ruzic-Sabljic E, Maraspin $\mathrm{V}$, Lotric-Furlan S, et al. Identification of three species of Borrelia burgdorferi sensu lato (B. burgdorferi sensu stricto, B. garinii and B. afzelii) among isolates from acrodermatitis chronica atrophicans lesions. J Invest Dermatol 1998;110:211-14.

3 Busch U, Hizoteufel C, Boehmer R, Fingerle V, Nitschko $\mathrm{H}$, Wilske B, Preac-Mursic V. Three species of Borrelia burgdorferi sensu lato (B. burgdorferi sensu stricto, B. afzelii and B. garinii) identified from cerebrospinal fluid isolates by pulsed-field gel electrophoresis and PCR. J Clin Microbiol 1996;34:1072-8.

4 Eiffert H, Karsten A, Thomssen R, Christen HJ. Characterization of Borrelia burgdorferi strains in Lyme arthritis. Scand J Infect Dis 1998;30:265-8.

5 Vasiliu V, Herzer P, Rössler D, Lehnert G, Wilske B. Heterogeneity of Borrelia burgdorferi sensu lato demonstrated by an ospA-type-specific PCR in synovial fluid from strated by an ospA-type-specific PCR in synovial fluid from
patients with Lyme arthritis. Med Microbiol Immunol patients with Lyme arth

6 Van Der Heijden I, Wilbrink B, Rijpkema SGT, Schouls LM, Heymans PHM, Van Embden JDA, et al. Detection of Borrelia burgdorferi sensu stricto by reverse line blot in the joints of dutch patients with Lyme arthritis. Arthritis Rheum 1999;42:1473-80.

7 Priem S, Lunemann JD, Zarmas S, Franz JK, Aberer E, Rittig MG, et al. Rapid subtyping of Borrelia burgdorferi species in bacterial cultures and clinical specimens using DNecies in bacterial cultures and clinical specimens using Arthritis Rheum 1999;42 (suppl 9):S338.

8 Jaulhac B, Heller R, Limbach FX, Sibilia J, Monteil H, Kuntz JL, et al. Direct molecular typing of Borrelia burgdorferi sensu lato species in synovial samples from patients with Lyme arthritis. J Clin Microbiol 2000;38: 1895-900.

9 Jaulhac B, Chary-Valckenaere I, Sibilia J, Javier RM, Piémont Y, Kuntz JL, et al. Detection of Borrelia burgdorferi by DNA amplification in synovial tissue samples from patients with Lyme arthritis. Arthritis Rheum 1996;39: patients

10 Strle F, Nadelman R, Cimperman J, Nowakowski J, Picken $\mathrm{RN}$, Schwartz I, et al. Comparison of culture-confirmed erythema migrans caused by Borrelia burgdorferi sensu stricto in New York State and by Borrelia afzelii in Slovenia. Ann Intern Med 1999;130:32-6.

11 Priem S, Burmester GR, Kamradt T, Wolbart K, Rittig MG, Krause A. Detection of Borrelia burgdorferi by polymerase chain reaction in synovial membrane, but not in synovial fluid from patients with persisting Lyme arthriis after antibiotic therapy. Ann Rheum Dis 1998;57:11821 .

12 Kalish RA, Leong JM, Steere AC. Association of treatmentresistant chronic Lyme arthritis with HLA-DR4 and antibody reactivity to OspA and OspB of Borrelia burgdorferi. Infect Immun 1993;61:2774-9.

13 Gross DM, Forsthuber T, Tary-Lehmann M, Etling C, Ito K, Nagy ZA, et al. Identification of LFA-1 as a candidate autoantigen in treatment resistant Lyme arthritis. Science 1998;281:703-6.

14 Chen J, Field JA, Glickstein L, Molloy PJ, Huber BT, Steere AC. Association of antibiotic treatment-resistant Lyme arthritis with $T$ cell responses to dominant epitopes of outer surface protein A of Borrelia burgdorferi. Arthritis Rheum 1999;42:1813-22. 